\title{
Lunar Volatiles Orbiters
}

A White Paper Submitted To

The Decadal Survey in Planetary Science and Astrobiology 2023-2032

Submitted By:

Paul G. Lucey ${ }^{1}$, P Prem ${ }^{2}$, Morgan L. Cable ${ }^{3}$, Charles A. Hibbitts ${ }^{2}$, Casey I.

Honniball ${ }^{4}$, Carlé M. Pieters ${ }^{5}$, Michael Barker ${ }^{4}$, Mehdi Benna ${ }^{4}$, Darby Dyar ${ }^{6}$, Elizabeth Fisher $^{5}$, Paul Hayne ${ }^{7}$, Shuai $\mathrm{Li}^{1}$, Molly McCanta ${ }^{8}$, Dana M. Hurley ${ }^{2}$, Erwan Mazarico ${ }^{4}$, Kathleen E. Mandt ${ }^{2}$, Noah E. Petro ${ }^{4}$, Thomas M. Orlando ${ }^{9}$, and William M. Farrell ${ }^{4}$.

\author{
${ }^{1}$ University of Hawai ${ }^{\top} i$ at Mānoa \\ ${ }^{2}$ Johns Hopkins University/Applied Physics Laboratory \\ ${ }^{3}$ NASA Jet Propulsion Laboratory, California Institute of Technology \\ ${ }^{4}$ NASA Goddard Space Flight Center \\ ${ }^{5}$ Brown University \\ ${ }^{6}$ Mount Holyoke/Planetary Science Institute \\ ${ }^{7}$ University of Colorado Boulder \\ ${ }^{8}$ University of Tennessee, Knoxville \\ ${ }^{9}$ Georgia Institute of Technology
}

*Contact: lucey@hawaii.edu; 808-221-2135. 


\section{Lunar Volatiles Orbiters}

Orbital measurements of the lunar surface and exosphere occupy a pivotal role in the understanding of the lunar volatile system, and by extension the interaction of airless surfaces with the space environment in our Solar System and beyond. Orbital measurements offer a global synoptic view that provides vital context for surface measurements, and can be crucial when not possible from surface platforms. In this white paper we address the unique contributions from the vantage point of lunar orbit to the science areas defined in the companion white paper by Prem et al. "Lunar Volatiles and Solar System Science," and complements the contribution by Hayne et al. "New Approaches to Lunar Ice Detection and Mapping" the latter of which goes into detail why and how water ice can be detected in the lunar polar regions.

What are the relative contributions of impacts, volcanism and solar wind to the lunar polar volatile inventory, and how have these sources varied over time?

Between $\sim 4$ to $\sim 1 \mathrm{Ga}$, volcanism was the dominant geological process occurring on the Moon (Head, 1976 provides a review). Magma brought volatiles to the lunar surface that degassed during ascent and eruption (Wilson and Head, 1981, 2018). Needham and Kring 2017 estimate that over three billion years of mare volcanism, with peaks in activity at 3.8 and $3.5 \mathrm{Ga}, \sim 10^{16} \mathrm{~kg}$ of $\mathrm{CO}$ and $\mathrm{S}$ and $\sim 10^{14} \mathrm{~kg}$ of $\mathrm{H}_{2} \mathrm{O}$ was released and just $0.1 \%$ of this $\mathrm{H}_{2} \mathrm{O}$ could account for the entirety of the $\sim 10^{11} \mathrm{~kg}$ of water-equivalent hydrogen estimated to be present in the upper meter of the subsurface at the lunar poles today. At the same time, the lunar surface underwent a period of heavy bombardment by the remnants of planetary accretion: leftover planetesimals, comets and asteroids -some catapulted from the outer to the into the inner solar system by dynamical instabilities generated as the giant planets transitioned to their present orbits (Gomes et al., 2005, Morbidelli et al., 2018). Basin-forming impactors and their lesser contemporaries will have delivered massive quantities of volatiles to the lunar environment, but preservation is dependent on poorly known time of the formation of the polar cold traps. Order-ofmagnitude estimates for water supply to lunar polar cold traps through cometary and asteroidal impacts over the last $1 \mathrm{Ga}$ are $\sim 6 \times 10^{12 \mathrm{~kg}}$. [Ong et al. 2010] and extending this to the upper Imbrian with increasing impact flux to the past yields extremely high water ice inputs to the poles, as much as a few kilometers.

The principal distinguishing characteristics of volatiles derived from impact versus volcanism are the relative abundances of water, carbon, nitrogen and sulfur-bearing compound. Volcanism produces little water relative to carbon or sulfur and lacks nitrogen, whereas impacts bring abundant water and carbon compounds, some nitrogen but little sulfur. Perhaps significantly, the LCROSS impact revealed that sulfur is the most abundant element by mass after oxygen, an abundance far higher than can be accounted for by cometary or asteroidal inputs alone if original ratios are even approximately maintained (Colaprete et al. 2010). There is also a hint of the presence of sulfur in laser reflectance data for the lunar poles, where there is an inflection in the curve of temperature and reflectance at the volatilization temperature of sulfur (Fisher et al. 2017). While a landed package could be extremely effective at detecting traces of elemental sulfur and its reduced and oxidized gases $\left(\mathrm{H}_{2} \mathrm{~S}\right.$ and $\left.\mathrm{SO}_{2}\right)$, polar conditions are 
highly variable in temperature and geologic history and so generalization is difficult, as in the case of LCROSS.

Lunar Trailblazer is well suited to detect water and ammonia ice (provided there is enough illumination for the latter), but does not cover the UV, visible and IR wavelengths needed for detection of the strongest absorptions of sulfur and its ices (although weak $\mathrm{SO}_{2}$ features are present within its wavelength range). However, $\mathrm{SO} 2$ and $\mathrm{H} 2 \mathrm{~S}$ have strong spectral features near 4 microns can be observed in reflected light in permanent shadow or by laser spectrometers. Furthermore icy ejecta expelled from the continuous small meteorite impacts detected by LDEX can be measured by orbital in situ mass spectroscopy. This leaves for the future a survey of sulfur compounds and possible assessment of the relative contributions of impact and volcanism.

Solar wind volatile inputs are far lower, approximately 5 meters of equivalent water ice to the poles in 3.8 by. However, as a continuous steady source, the relative contribution of the solar wind depends critically on the timing of the onset of cold trapping, and has a distinct isotopic signature, with vanishingly small $\mathrm{D} / \mathrm{H}$ ratios due to consumption of deuterium in the Sun. Methane has been detected in the lunar atmosphere by Hodges (2016) attributed to solar wind carbon and hydrogen; this compound will cold trap in the PSR at the few tens of ppm level, may contribute feedstock to on-going organic reactions and will be isotopically distinct from cometary organics in $\mathrm{D} / \mathrm{H}$.

Orbital remote sensing has the potential to distinguish volcanic from impact sources, but existing and planned missions have little capability to detect the presence of sulfur and its compounds which is critical to testing the relative contributions these ancient sources. The contribution of solar wind is indicated by $\mathrm{D} / \mathrm{H}$ ratios owing to the extremely low ratios of this source. D/H can be measured for ice "dust" with an orbital mass spectroscopic dust detector with suitable mass resolution.

\section{What are the transport, retention, alteration, and loss processes that operate} on volatiles in the lunar polar environment?

The contemporary lunar volatile system is by definition dynamic, and includes inputs from the solar wind and meteorites, processing in the lunar surface, transport to the polar regions, loss to space enroute, deposition in the polar regions, and further processing, sequester and loss in the polar regions.

The orbital platform contribution to global atmospheric dynamics is addressed below, but the polar surfaces must be dynamic as well. Volatiles arriving in the polar regions through exospheric transport from lower latitudes can be trapped in transient cold traps at the diurnal, seasonal or precessional timescales as well as the permanent cold traps. Nighttime surface abundances of mobile water or methane on the equatorial lunar surface are likely to be far below any current instrument limit of detection, but at the seasonal and precessional timescale, polar surfaces can accumulate volatiles for on the order of 10 to 100 times longer than the diurnal scale, respectively. When those surfaces are illuminated they will promptly degas causing both a spectral change at the surface, and pulses of volatiles to the polar exosphere. The seasonal cycle of Ar abundance observed at the equator by LADEE may reflect pole to pole transport as the area of 
temporary cold traps varies seasonally, showing that variation in surface abundance of volatiles in the cold traps must be occurring.

Impact vaporization is an energetic process that leads to escape of most of the released molecules from the vicinity of the impact, even the smallest. These water molecules either are lost to space $(\mathrm{v}>2.4 \mathrm{~km} / \mathrm{s})$ or ballistically land typically a few hundred $\mathrm{km}$ from the target site $(\mathrm{v}<2.4 \mathrm{~km} / \mathrm{s})$ [Farrell et al., 2015] where they are subject to further migration if they do not land in a low temperature PSR. The particulates associated with meteoric impact ejection are launched at lower velocities and a substantial fraction $(\sim 10 \%)$ will ballistically fall within a PSR upon their release [Farrell et al., 2019]. Some of the particulates will land outside PSR where their volatiles can remobilize, some to return to PSRs.

On surfaces below about $100 \mathrm{~K}$, sublimation is negligible so the effectiveness of other loss mechanisms can be explored. Plasma sputtering by solar wind diverted into PSR by ambipolar electric fields is highly dependent on topography and the latitude and longitude of the PSR, so the correlation of maximum sputtering effectiveness can be explored through comparison of measured distributions with modeled sputtering intensity (Farrell et al., 2010; Zimmerman et al., 2011).

In the case of water ice, measurements near 3.1 microns are sensitive to a few tens of nm of changes in ice thickness, and sputtered ions from these temporary ice surfaces will be launched into the exosphere, so the effect of surface volatile deposition on transient cold traps, or impact redistribution of volatiles in PSRs may be observable.

Global orbital remote sensing has the potential to detect time variations in the surface abundance of water ice and other volatiles that will reflect the dynamism of the polar regions due to impact and diurnal, seasonal and precessional timescales.

\section{What is the distribution and physical form of lunar surface volatiles beyond}

the poles?

The chemical nature of the hydrogen compound detected on the lunar surface is unknown. Based principally on the temperature-dependent variation in the strength of the observed $3 \mu \mathrm{m}$, Sunshine et al. [2009] concluded the infrared spectral variations were due to molecular water migrating along temperature gradients on the lunar surface. However, measurements at $3 \mu \mathrm{m}$ cannot distinguish molecular water $\left(\mathrm{H}_{2} \mathrm{O}\right)$ from hydroxyl bonded to a metal cation $(\mathrm{M}-\mathrm{OH})$. The presence of molecular water was suggested by Hendrix et al. [2012; 2019] as the LRO/LAMPUV spectrometer revealed a diurnal variation in UV absorption where the UV absorption decreases substantially between 10 to 14 hours local time (near local noon) when the surface is the warmest. They suggest the LAMP data indicates a migratory pattern of long-lived, hopping water that is effectively avoiding/hopping away from the warm regions. While this agrees generally with Sunshine et al. [2009] using IR data, it is in conflict with observations of the exospheric water density by LADEE/NMS. Furthermore, as pointed out by Hendrix et al. [2012], there are no FUV spectral data on adsorbed water or hydroxyl. Therefore, the detection of water or hydration using LAMP data is based on the hypothesis that hydration features in 
the FUV are qualitatively and quantitatively similar to that of water ice.

If molecular water is not present, and hydroxyl is varying in the lunar surface, this has implications for long term sequestration of hydroxyl in the regolith and availability for production of water during meteoritic impact as hydroxyl cannot migrate in significant quantities. To explain hydroxyl variation, Farrell et al. [2017], and Tucker et al. [2019] suggest based on the work of Starukhina [2006] and Fink et al. [1995] that H atoms are will migrate from oxygen to oxygen within the grain and form metastable $\mathrm{OH}$ near damaged $\mathrm{O}$ in the surface as a function of temperature. This means the hydroxyl signal does not represent long-term storage, but rather is a byproduct of rapid escape of implanted hydrogen. In contrast, Jones et al. [2018] and McCord et al. [2011] suggest solar wind protons are implanting and producing stable $\mathrm{OH}$ groups in the top $100 \mathrm{~nm}$ of the lunar surface. The critical discriminator is true diurnal variation in hydroxyl [and water? clarify]. In the absence of molecular water, diurnal variations suggest weak retention of $\mathrm{OH}$; weak or absent diurnal variations indicate the presence of long-lived metal-OH bonds.

However, the existence of variation in the abundance of hydroxyl or water based on spectral variation has been called into question. Reflectance data near $3 \mu \mathrm{m}$ are contaminated by lunar thermal emission, and results are critically dependent on how these terms are separated. Three major studies of the variation of hydroxyl, all using the same data from Chandrayaan-1 M3, reached different conclusions. Li and Milliken [2017] found a strong latitudinal variation in hydroxyl, with little to no hydroxyl detected below $\sim 25$ degrees latitude. Between $\sim 25$ and $\sim 65$ degrees they found a strong (up to $\sim 100 \mathrm{ppm}$ ) diurnal effect that is somewhat asymmetric between morning and afternoon, but little variation above $\sim 65$ degrees. In contrast, Wohler et al. [2017] and Grumpe et al. [2019] suggest weak latitudinal variation and no diurnal variation below $\sim 30$ degrees, but strong diurnal variation from $\sim 30$ degrees to the pole that is symmetric between morning and afternoon. Bandfield et al. [2018] find no variation with time-of-day, temperature, or latitude, but do agree that a hydroxyl band is present. All these studies use similar physics, but subtly different assumptions regarding photometric and subpixel temperature behavior. This brings into sharp focus the need to bring new approaches including using laser spectroscopy to detect true variations in hydroxyl abundance, and spectroscopy at 6 $\mu \mathrm{m}$ (e.g. Honniball et al. 2020) to definitively detect, or rule out, molecular water on the surface.

Finally, other chemistry is occurring in the lunar surface. The mineral hematite-oxidized iron--was recently discovered at high latitudes in $\mathrm{M}_{3}$ observations of the illuminated surface [Li et al., 2019], suggested to be due to interaction of lunar iron with terrestrial oxygen or migrating lunar water. Furthermore, based on the detection of methane in the lunar exosphere, Hodges [2016] suggests solar wind hydrogen and carbon are implanting, diffusing, and concentrating within the top $\sim 100 \mathrm{~nm}$ of the surface to synthesize hydrocarbons. Lunar hydrocarbons may have been detected in samples of lunar micrometeorite impact glass by Liu et al. [2012], and high-albedo organic species (such as ethane, HCN, methanol and formate) may be the first products of organic synthesis in ice deposits in the PSR, as lunar radiation may be too weak for albedolowering carbonization as on Mercury [Delitsky et al. 2017].

Orbital remote sensing with new instrumentation can definitively characterize the behavior of water and hydroxyl on the surface, and extend the limit of understanding of lunar surface chemistry to better target landed packages for more detailed analysis. 
Early studies assumed cold trapping was the only mechanism for stopping a water molecule on interaction with the surface (Watson et al. 1961, Butler et al. 1993, Arnold, 1979). Unlimited migration offers a persistent supply of water to the poles from anywhere on the Moon where water is introduced. But during its travels, water may stick to the surface either temporarily or permanently, potentially severely limiting the capture region for polar water. If adsorptive sites are present on the surface that exceed the thermal energy available to the molecule, many will be trapped and only a fraction may migrate. Molecular water may also dissociatively adsorb (Gun'ko et al., 1998), that is, break into a hydroxyl and hydrogen and no longer migrate. The exospheric density of water with longitude and time of day is strongly influenced by the ability of water to migrate. With unlimited migration, water abundance strongly peaks near the dawn terminator on the day side, with its time of day maximum dictated by activation energy. This occurs because water molecules that are activated at dawn can jump to the night side of the terminator and be quickly reactivated by the advancing dawn terminator producing a "snowplow" effect [Schorghofer, 2014]. No equivalent buildup occurs at the retreating evening terminator. However, if water migration is minimal, the distribution of exospheric density is much flatter, and with maximum near the source time of day maximum. This time of day spatial distribution of water adsorbed to the surface is a signature of the migrating water scenario and precise exospheric measurements of background water could directly test this. The absolute exospheric abundance is also an indicator of the degree of migration, as for a given source intensity, multiple hops will populate the atmosphere roughly proportional to the average number of hops.

Hurley et al. [2019] recently found that if $\sim 10^{-4}$ of the incoming solar wind protons are converted to water at low latitudes, the resulting exospheric density of the water (onehop or multi-hop) is $\sim 1-5 / \mathrm{cm}^{3}$ - at $50 \mathrm{~km}$, well within the detection limit of neutral mass spectroscopy instruments. Sensitivity of $0.1 / \mathrm{cc}$ and time resolution of 2 hours local time is more than sufficient to distinguish these model cases based both on the shape of the diurnal curve (either sharply peaking at dawn or showing a flatter distribution), and the absolute exospheric abundance. LADEE upper limits on absolute exospheric density suggest no hopping of water occurs (Hurley et al. [2019]); precise exospheric water measurements would determine the shape of the exospheric density curve with time of day, greatly increasing robustness of the estimate.

Finally, water and other molecules will sorb or 'stick' to a surface. The release or desorption of the molecule requires energy ("activation energy of desorption) in order for the molecule to be liberated from the atom-to-atom molecular bond. Surface or orbital measurements can be used to estimate this critical parameter. UV observations have estimated the activation energy to liberate water from the lunar surface. Hendrix et al. [2012; 2019] suggested that the LRO/LAMP UV spectrometer revealed a diurnal variation in molecular water where the signature of hydration decreases substantially between 10 to 14 hours local time (near local noon) - where the surface is the warmest. They suggest that the LAMP data is indicating a migratory pattern of long-lived hopping water (that is effectively avoiding/hopping away from the warm regions). Using this migration pattern, Hendrix et al. [2019] suggest that the surface activation energy for the 
bound water is high, near $1.2 \mathrm{eV}$ - with migration initiated only at the highest temperatures in mid-day regions. However, the diurnal pattern observed in the IR shows release at lower temperatures, if attributed to molecular water and implies a lower activation energy. Both of these observations are plagued by the ambiguity between hydroxyl and water.

There are at least five pathways for incident solar wind protons $\left(\mathrm{p}^{+}\right)$to escape the surface: $(1) \sim 1-2 \%$ of the incoming protons are immediate backscattered into the solar wind as energetic protons, $\mathrm{p}^{+}$, with energies between $100 \mathrm{eV}$ and $1 \mathrm{keV}$ [Saito et al., 2008]. (2) About 7-35\% of the incoming solar wind proton flux is immediately backscattered as energetic neutral $\mathrm{H}$ between $<30-1000 \mathrm{eV}$ [McComas et al., 2009; Futaana et al., 2012; Vorburger et al., 2012]. (3) There is thermal $\mathrm{H}_{2}$ predicted by Starukhina [2006] and observed by LAMP [Hurley et al., 2017], with 10-50\% of the incoming solar wind ions converting to a molecular form. (4) LADEE NMS observed $\mathrm{CH}_{4}$, suggesting that solar wind protons and carbon are forming and releasing $\mathrm{CH}_{4}$ in the top surface of the lunar regolith [Hodges, 2016]. (5) The observation of energetic neutral $\mathrm{H}$ atoms also points to the strong possibility of a thermal $\mathrm{H}$ escape process that has yet to be measured [Hodges, 2011]. There could thus be a thermal H corona about the Moon the gravity binding being very weak making it a 'leaky' exosphere. The fraction of thermal $\mathrm{H}_{2}$ vs $\mathrm{H}$ is currently not known.

The integrated picture is that the lunar surface 'breathes' hydrogen, taking in solar wind protons, retaining them in cool regions and releasing them as new $\mathrm{H}$ chemical products the surface warms. Given the understanding of the lunar hydrogen cycle, we can extend our understanding for the system at Mercury, Phobos, S-class asteroids and all bodies directly exposed to the solar wind.

An integrated approach of measuring global exospheric water and surface hydration from orbit can determine the relative contributions of meteorites and solar wind to the contemporary system, and define the mobility of water and hydroxyl.

\section{The Next Decade}

The most pressing measurement campaign to be made is a global polar survey of water in the lunar exosphere to the background imposed by sporadic wet meteors. The lunar water atmosphere will be overwhelmed by the operations of planned large spacecraft and may nullify the use of the lunar environment to test competing hypotheses.

A full accounting of the lunar hydrogen cycle will enable extension to airless object in the Solar System and exoplanets exposed to stellar winds.

Active spectroscopy may be required to resolve the issue of possible mobile surface water or hydroxyl, and the same capability can measure extremely small quantities of water ice and potentially detect dynamics in the polar regions. The coldest locations hosting supervolatiles such as $\mathrm{CO} 2$ may require active spectroscopy owing to limited available light.

High spectral resolution is needed to identify organic species to determine the potential for organic chemistry in the PSR. 
References: Benna, M., Hurley, D., Stubbs, T., Mahaffy, P., Elphic, R., 2019. Nature Geoscience 12, 333; Bandfield, J.L., Poston, M.J., Klima, R.L., Edwards, C.S., 2018. Nature geoscience 11, 173; Colaprete, A., et al. 2010. Science, 330(6003), p. 463-468; Delitsky, M. L., et al. (2017). Icarus, 281, 19-31; Dyar, M., Hibbitts, C., Orlando, T., 2010. Icarus 208, 425-437; Farrell WM, Hurley DM, Zimmerman MI. GRL. 2015 May 16;42(9):3160-5; Farrell, W., Hurley, D., Esposito, V., McLain, J., Zimmerman, M., 2017. JGR 122, 269-289; Farrell WM, Hurley DM, Poston MJ, Hayne PO, Szalay JR, McLain JL.. GRL; 2019 Aug 16;46(15):8680-8; Farrell, W. M., T. J. Stubbs, J. S. Halekas, R. M. Killen, G. T. Delory, M. R. Collier, and R. R. Vondrak (2010), JGR., 115, E03004, doi:10.1029/2009JE003464; Fink, D., et al. (1995) , Appl. Phys. A, 61, 381-388; Fisher, E. A., et al. . (2017). Icarus, 292, 74-85; Futaana, Y., et al. (2012), JG., 117, E05005, doi:10.1029/2011JE004019; Gomes R., Levison H.F., Tsiganis K., and Morbidelli A. (2005) Nature 435, 466-469; Grumpe, A., Wöhler, C., Berezhnoy, A.A., Shevchenko, V.V., 2019. Icarus 321, 486-507; Gun'ko, V., et al. 1998. International journal of mass spectrometry and ion processes 172, 161-179; Hayne, P. O., Hendrix, A., Sefton-Nash, E., Siegler, M. A., Lucey, P. G., Retherford, K. D., ... \& Paige, D. A. (2015). Icarus, 255, 58-69; Head III, J. W., 1976. Rev. Geophys, 14(2), p. 265-300; Hendrix, A.R., et al. 2012. JGR 117; Hendrix, A.R., Hurley, D.M., Farrell, W.M., Greenhagen, B.T., Hayne, P.O., Retherford, K.D., Vilas, F., Cahill, J.T., Poston, M.J., Liu, Y., 2019. GRL 46, 2417-2424; Hodges, R.R., (2011). GRL. 38, L06201. http://dx.doi.org/10.1029/ 2011GL046688; Hodges Jr RR. GRL. 2016 Jul 16 43(13):6742-8; Honniball C.I, et al.. LPI. 2020 Mar(2326):1422; Hurley, D., Prem, P., Benna, M., Vondrak, R., Farrell, W., Hendrix, A., Lucey, P., 2019. LPI 2019; Hurley, D. M., J. C. Cook, K. D. Retherford, et al. (2017), Icarus, 283, 31-37; Jones, B.M., Aleksandrov, A., Hibbitts, K., Dyar, M., Orlando, T.M., 2018. GRL 45, 10,959-910,967; Li, S., Milliken, R.E., 2017. Science advances 3, e1701471; Li S, Lucey PG, Fraeman A, Poppe AR, Sun VZ, Hurley D, Schultz PH. AGUFM. 2019 Dec;2019:P54C-04; Liu, Y., Guan, Y.B., Zhang, Y.X., Rossman, G.R., Eiler, J.M., Taylor, L.A., 2012. Nature Geoscience 5, 779-782; McComas, D. J., et al. (2009), GRL 36, L12104, doi:10.1029/2009GL038794; McCord, T.B., Taylor, L.A., Combe, J.P., Kramer, G., Pieters, C.M., Sunshine, J.M., Clark, R.N., 2011. JGR-Planets 116; Milliken R.E. and Li S. (2017) Nature Geoscience 10, 561-565; Morbidelli A., Levison H.F., Tsiganis K. and Gomes R. (2005) Nature 435, 462-465; Needham, D. H. and Kring, D. A., 2017. EPSL, 478, p. 175-178; Ong L, Asphaug EI, Korycansky D, Coker RF. Icarus. 2010 Jun 1;207(2):578-89; Pieters, C.M., et al.., 2009. Science 326, 568-572; Pokorný P, Sarantos M, Janches D, Mazarico E. The Astrophysical Journal. 2020 May 12;894(2):114; Ruff, S. 2004. Icarus, 168:1, 131-143; Saito, Y. et al., (2008), GRL. 35, L24205. http://dx.doi.org/10.1029/ 2008GL036077; Schorghofer, N., 2014. GRL 41, 4888-4893; Starukhina, L. V. (2006). Advances in Space Research, 37(1), 50-58. https: //doi.org/10.1016/ j.asr.2005.04.03; Sunshine, J.M., Farnham, T.L., Feaga, L.M., Groussin, O., Merlin, F., Milliken, R.E., A'Hearn, M.F., 2009. Science 326, 565-568; Szalay, J. R., Pokorny', P., Sternovsky, Z., Kupihar, Z., Poppe, A. R., \& Horányi, M. (2019). JGR: Planets, 124. https://doi.org/10.1029/ 2018JE005756; Tucker, O., Farrell, W., Killen, R., Hurley, D., 2019.. JGR 124, 278-293; Vorburger, A., et al. (2012), JGR 117, A07208, doi:10.1029 /2012JA017553; Watson, K., Murray, B.C., Brown, H., 1961. JGR 66, 3033-3045; Wilson, L. and Head III, J. W., 1981. JGR: Solid Earth, 86(B4), p. 2971-3001; Wilson, L. and Head, J. W., 2018. GRL, 45(12), p. 5852-5859; Wöhler, C., Grumpe, A., Berezhnoy, A.A., Shevchenko, V.V., 2017. Science advances 3, e1701286; Zhang JA, Paige DA. GRL. 2009 Aug;36(16); Zimmerman, M. I., Farrell, W. M., Stubbs, T. J., Halekas, J. S., \& Jackson, T. L. (2011). GRL, 38, L19202. https://doi.org/10.1029/2011GL048880 